\title{
Stringent response and initiation of secondary metabolism in Streptomyces clavuligerus
}

\author{
Victoria Bascarán, Lourdes Sánchez, Carlos Hardisson and Alfredo F. Braña* \\ Laboratorio de Microbiología, Departamento de Biología Funcional, Facultad de Medicina, Universidad de Oviedo, \\ 33006 Oviedo, Spain
}

(Received 18 September 1990; revised 28 January 1991; accepted 28 February 1991)

\begin{abstract}
Cephalosporin biosynthetic activity and extracellular protease production increased during growth of Streptomyces clavuligerus in defined medium, while the level of guanosine $5^{\prime}$-diphosphate 3 -diphosphate (ppGpp) remained very low and stable. Cephalosporin biosynthesis (measured in resting cell systems) was initiated during early exponential growth in complex media, without appreciable change in the small ppGpp pool. Nutritional shiftdown induced by withdrawal of Casamino acids caused a transient increase in ppGpp and a reduction of RNA accumulation. The increase in ppGpp was small in very young cultures, but increased as the culture aged. Twentyseven spontaneous thiostrepton-resistant mutants were isolated and partially characterized. Most of them had a reduced ppGpp-forming ability and gave normal titres of cephalosporin. However, in complex medium, some mutants did not produce cephalosporins or extracellular protease, whereas others overproduced cephalosporins. The results indicate that, in $S$. clavuligerus, there is no obligatory relationship between the initiation of secondary metabolism and the stringent response.
\end{abstract}

\section{Introduction}

Streptomyces species synthesize a great variety of antibiotics and other secondary metabolites. A single strain is often capable of producing several chemically unrelated compounds. These biosynthetic activities seem to be regulated by at least two types of control. Signals generated by the nature and concentration of specific nutrients (mainly carbon, nitrogen or phosphorus compounds) determine the functioning of particular pathways. Thus a strain producing several secondary metabolites can synthesize such products either simultaneously or separately (Bushell \& Fryday, 1983; Romero et al., 1984; Hobbs et al., 1990), and pleiotropic mutants blocked in secondary metabolism and, sometimes, morphological differentiation, have been isolated. In Streptomyces coelicolor, several known master genes are responsible for these pleiotropic effects (Hopwood, 1988; Adamidis et al., 1990). This implies that secondary metabolism and differentiation are hierarchically regulated, and suggests the possible existence of a metabolic signal that would represent the first step towards the switching on of both processes.

Abbreviations: DAT, diamine aminotransferase; DCW, dry cell weight; LAT, lysine $\varepsilon$-aminotransferase; RCA, resting cell activity; TSB, Tryptone Soya Broth.
Work on several Streptomyces strains has implicated the stringent response as an initiating signal (Ochi, 1986, $1987 a, b, 1988)$. In these strains, antibiotic production was initiated after nutritional shift-down and increased guanosine $5^{\prime}$-diphosphate $3^{\prime}$-diphosphate (ppGpp) and guanosine $5^{\prime}$-triphosphate $3^{\prime}$-diphosphate (pppGpp) synthesis. Some spontaneous thiostrepton-resistant mutants with different alterations in their ribosomal protein patterns (Ochi, 1990) formed less ppGpp under shiftdown conditions, and were impaired in antibiotic production. Furthermore, ppGpp could be indirectly involved in morphological differentiation as an inhibitor of GTP synthesis (Ochi, 1986, $1987 a, c$ ), in a way similar to that proposed for sporulation in Bacillus (Freese et al., 1985). In other studies where ppGpp levels were measured during the onset of antibiotic production, a correlation was found in Streptomyces aureofaciens (Šmúth et al., 1979), but not in Streptomyces griseus (An \& Vining, 1978).

We have investigated the possible involvement of ppGpp in the initiation of secondary metabolism in Streptomyces clavuligerus. This micro-organism produces several different antibiotics (cited in Romero et al., 1984), as well as an extracellular metalloprotease with regulatory behaviour suggesting a partial linkage with antibiotic formation (Bascarán et al., 1990). Therefore, 
this strain offers the opportunity to distinguish signals that affect a single secondary metabolic activity from possible hierarchical signals.

\section{Methods}

Organisms. Wild type Streptomyces clavuligerus NRRL 3585 (ATCC 27064) was used. Spontaneous thiostrepton-resistant mutants were isolated on plates with sporulation medium (Braña et al., 1985) supplemented with $3 \mu \mathrm{g}$ thiostrepton $\mathrm{ml}^{-1}$. Colonies obtained after $30 \mathrm{~d}$ incubation at $28^{\circ} \mathrm{C}$ were purified and maintained in the same medium in the presence of 3 or $5 \mu \mathrm{g}$ thiostrepton $\mathrm{ml}^{-1}$. Growth of the wild-type strain was completely inhibited by $1 \mu \mathrm{g}$ thiostrepton $\mathrm{ml}^{-1}$.

Media and culture conditions. S. clavuligerus was grown in liquid media based on a synthetic medium (MF medium) that contained per litre of water: glycerol, $10 \mathrm{~g} ; \mathrm{K}_{2} \mathrm{HPO}_{4}, 3.5 \mathrm{~g} ; \mathrm{MgSO}_{4} .7 \mathrm{H}_{2} \mathrm{O}, 0.6 \mathrm{~g}$; $\mathrm{FeSO}_{4} .7 \mathrm{H}_{2} \mathrm{O}, 5 \mathrm{mg} ; \mathrm{MnCl}_{2} .4 \mathrm{H}_{2} \mathrm{O}, 5 \mathrm{mg} ; \mathrm{ZnSO}_{4} . \mathrm{H}_{2} \mathrm{O}, 5 \mathrm{mg} ; \mathrm{CaCl}_{2}$, $5 \mathrm{mg}$. This medium was buffered with MOPS and adjusted to $\mathrm{pH} 6.9$ with $8 \mathrm{M}-\mathrm{KOH} ; 25 \mathrm{mM}-\mathrm{MOPS}$ was used for incubations stopped in mid-growth phase, and $100 \mathrm{mM}-\mathrm{MOPS}$ for incubations continued up to stationary phase. Trace elements were added as previously described (Bascarán et al., 1989) but the concentration was increased from 1 to $5 \mathrm{mg} \mathrm{1}^{-1}$. This modification increased protease titres three- to fourfold over those obtained in batch cultures by Bascarán et al. (1990). Nitrogen sources were added as described in the text. Concentrated spore suspensions (approximately $5 \times 10^{9}$ spores $\mathrm{ml}^{-1}$ ) were prepared by scraping off the spores obtained on sporulation medium and suspending them in $50 \%(\mathrm{w} / \mathrm{v})$ glycerol in $50 \mathrm{~mm}-\mathrm{Tris} / \mathrm{HCl}, \mathrm{pH} 7 \cdot 2$. The suspensions were sonicated in an ice-cold ultrasonic bath for $5 \mathrm{~min}$ and kept at $-70^{\circ} \mathrm{C}$. Unless otherwise indicated, cultures were inoculated with mycelium grown for $48 \mathrm{~h}$ in seed medium inoculated with spores (Aharonowitz \& Demain, 1979). Culture conditions, growth determinations and strain maintenance were as described previously (Bascarán et al., 1989). In experiments requiring precise growth measurements, the flasks were siliconized with Sigmacote (Sigma), a treatment that prevented wall growth. In shift-down experiments, cells were collected on a membrane filter and washed with nitrogen-free MF medium, and the filter with the cells was immediately immersed in the new medium, which had been prewarmed to $28^{\circ} \mathrm{C}$.

Cephalosporin production. Cephalosporins, mainly cephamycin C (Braña et al., 1985) were measured by bioassay with Escherichia coli ESS as the indicator micro-organism, and cephalosporin $\mathrm{C}$ as the standard (Braña et al., 1985). For the assay of samples containing chloramphenicol, a chloramphenicol-resistant derivative, E. coli ESSC (donated by Y. Aharonowitz, Tel-Aviv University, Israel), was the indicator strain. One unit (U) of antibiotic is defined as the amount producing an inhibition zone equivalent to $1 \mu \mathrm{g}$ cephalosporin $\mathrm{C}$.

Resting cell systems. The antibiotic-synthesizing ability of the cells was determined at selected times in washed mycelial suspensions incubated for $2 \mathrm{~h}$ in $0 \cdot 1 \mathrm{M}$-MOPS buffer, $\mathrm{pH} \mathrm{6.8,} \mathrm{containing} 100 \mu \mathrm{g}$ chloramphenicol $\mathrm{ml}^{-1}$ (Braña et al., 1985). Initial dry cell weight (DCW) of the suspensions was adjusted to $8 \mathrm{mg} \mathrm{ml}^{-1}$. Resting cell activity (RCA) is defined as units of antibiotic produced per $\mathrm{mg} \mathrm{DCW}$ per hour under these conditions.

Enzyme assays. Extracellular protease was assayed with azocasein (Sigma) as the substrate (Ginther, 1979). One $U$ of activity is defined as the amount of enzyme that produces an increase in absorbance of 1.0 in $1 \mathrm{~h}$. Lysine $\varepsilon$-aminotransferase (EC 2.6.1.36) (LAT) and diamine aminotransferase (EC 2.6.1.29) (DAT) were determined in crude extracts obtained by ultrasonic disruption in $10 \mathrm{mM}-\mathrm{MOPS}, \mathrm{pH} 7.2$, containing $1 \mathrm{~mm}-\mathrm{MnCl}_{2}$ (Bascarán et al., 1989). Activities were estimated by spectrophotometric determination of the reaction products derivatized with $o$-aminobenzaldehyde (Sigma), as described by Madduri et al. (1989). One $U$ of activity is defined as the amount of enzyme that produces $1 \mu \mathrm{mol}$ product $\mathrm{min}^{-1}$. Protein in crude extracts was measured by the Lowry method.

RNA accumulation. Cultures with an approximate cell density of $1 \mathrm{mg}$ DCW $\mathrm{ml}^{-1}$ were labelled with $20 \mu \mathrm{M}-[2-14 \mathrm{C}]$ uracil $\left(1.95 \mathrm{kBq} \mathrm{ml}^{-1}\right)$ (New England Nuclear). Radiactivity incorporated into acid-precipitable material was determined at different times in $1 \mathrm{ml}$ samples, as described by Hardisson et al. (1978).

Nucleotide pools. Culture fractions containing $20 \mathrm{mg}$ DCW were collected on membrane filters $(47 \mathrm{~mm}$ diameter, $0.45 \mu \mathrm{m}$ pore size, Geiman Sci.) and extracted with $5 \mathrm{ml} 1 \mathrm{M}$-formic acid (Ochi, 1986). The collection procedure took less than $10 \mathrm{~s}$. After lyophilization of the samples, nucleotides were separated by HPLC on a Partisil SAX radial compression cartridge $(0.8 \times 10 \mathrm{~cm} ;$ Waters $)$. Elutions were carried out as described by Ochi et al. (1981), and column conditioning and regeneration according to Freese et al. (1984). Nucleotides were quantified by comparison of peak areas of standards, and the results are given as pmol per mg DCW extracted.

Nutrient consumption. Residual asparagine in the culture medium was determined by HPLC (Hill et al., 1979). Ammonium was measured by the phenol/hypochlorite method (Weatherburn, 1967). Residual glycerol was measured colorimetrically (Bok \& Demain, 1977).

Chemicals. Thiostrepton was a gift from S. Lucania, E. R. Squibb \& Sons, Princeton, New Jersey. Vitamin-free Casamino acids were purchased from Merck, ppGpp from Calbiochem AG, pppGpp from Pharmacia, and Tryptone Soya Broth (TSB) from Oxoid.

Reproducibility. Results given in Tables are the means of at least two independent experiments. The highest SD values, observed in measuring LAT activities and extracellular protease titres, were within $\pm 20 \%$ of the mean. Figs. 1-5 show representative results from single experiments, all of which were repeated at least twice with simultaneous or separate determinations of the parameters. Data in Figs. 6 and 7 are from a single screening, but the phenotype of mutants that produced less than $5 \mathrm{U} \mathrm{ml}^{-1}$ or more than $100 \mathrm{U} \mathrm{ml}^{-1}$ of cephalosporins under those conditions was confirmed in at least two additional experiments.

\section{Results}

\section{Nitrogen control of lysine catabolic enzymes}

The DAT and LAT activities obtained with different nitrogen sources are shown in Table 1. DAT levels were highest with lysine, and decreased in nitrogen-repressing conditions (i.e. with ammonium or glutamine plus ammonium; Bascarán et al., 1989). LAT was not induced by lysine, and its activity was maximal in media that supported the highest cephalosporin production.

\section{Expression of secondary metabolism in defined medium}

A series of parameters determined in MF medium with asparagine as the nitrogen source are shown in Fig. 1. Data on nutrient consumption indicated that nitrogen limited growth in this medium; asparagine was exhausted first, followed by the small amount of ammonium 
Table 1. Influence of the nitrogen source on DAT and LAT activities

\begin{tabular}{lccc}
\hline \hline & \multicolumn{2}{c}{$\begin{array}{c}\text { Specific activity } \dagger \\
\left.\text { [mU (mg protein })^{-1}\right]\end{array}$} \\
\cline { 2 - 4 } \multicolumn{1}{c}{ Nitrogen source* } & DAT & LAT & $\begin{array}{c}\text { Cephalosporins; } \\
\left(\mathrm{U} \mathrm{ml}^{-1}\right)\end{array}$ \\
\hline Lysine & 1.08 & 0.07 & 1.7 \\
Lysine $+\mathrm{NH}_{4} \mathrm{Cl}$ & 0.73 & $0 \cdot 12$ & 15.5 \\
Lysine $+\mathrm{NH}_{4} \mathrm{Cl}+$ glutamine & 0.45 & 0.66 & 21.0 \\
$\mathrm{NH}_{4} \mathrm{Cl}$ & 0.20 & 0.27 & 12.0 \\
Alanine & 0.36 & 0.18 & 15.0 \\
Asparagine & 0.30 & 0.81 & 33.0 \\
\hline \hline
\end{tabular}

* Each nitrogen source was used at a concentration providing $30 \mathrm{~mm}$ nitrogen.

† Determined in late growth phase (approximately $2 \mathrm{mg}$ DCW $\mathrm{ml}^{-1}$ ).

$\ddagger$ Maximum titre achieved in the fermentation.

(0.54 mM) generated during growth. Glycerol $\left(1.4 \mathrm{~g} \mathrm{l}^{-1}\right.$ at the time of ammonium depletion) was exhausted last. Phosphate concentration (initially $20 \mathrm{mM}$ ) does not limit growth in this medium (Aharonowitz \& Demain, 1977). Cephalosporins and metalloprotease were produced during growth, starting at an early phase. LAT was also detected early in growth, and reached a maximum immediately before the total exhaustion of nitrogen. Among the nucleotides measured, ATP levels showed the greatest variation (Fig. $1 b$ ). The pool of ppGpp remained constant at basal levels [less than 4 pmol (mg DCW $)^{-1}$ ] until the culture reached stationary phase. Single measurements of $\mathrm{ppGpp}$ in cells growing in MF medium with different nitrogen sources when the cultures were at $1.0 \pm 0.2 \mathrm{mg} \mathrm{DCW} \mathrm{ml}{ }^{-1}$ gave the following values [pmol $\left.(\mathrm{mg} \mathrm{DCW})^{-1}\right]:<3 \cdot 0$ for glutamate and glutamine, 5.4 for alanine, 6.5 for arginine, and 12.6 for proline. These ppGpp levels show an inverse correlation with the growth rate obtained in every case (Bascarán et al., 1990). pppGpp could not be detected $\left[<3 \mathrm{pmol}(\mathrm{mg} \mathrm{DCW})^{-1}\right]$ in any of these experiments.

\section{Initiation of secondary metabolism in complex medium}

MF medium with asparagine and $1 \%(w / v)$ Casamino acids was inoculated with a seed culture to obtain an initial DCW of $0.03 \mathrm{mg} \mathrm{ml}^{-1}$. The culture grew exponentially at first, and then at a gradually declining growth rate (Fig. $2 a$ ). RCA began to rise in the exponential phase. LAT and cephalosporins were detected sequentially, increasing in the course of the experiment. No extracellular protease was produced in this medium during the period considered. The ATP pool increased dramatically during early growth, while ppGpp remained below 3 pmol (mg DCW) ${ }^{-1}$ (Fig. $2 b$ ).
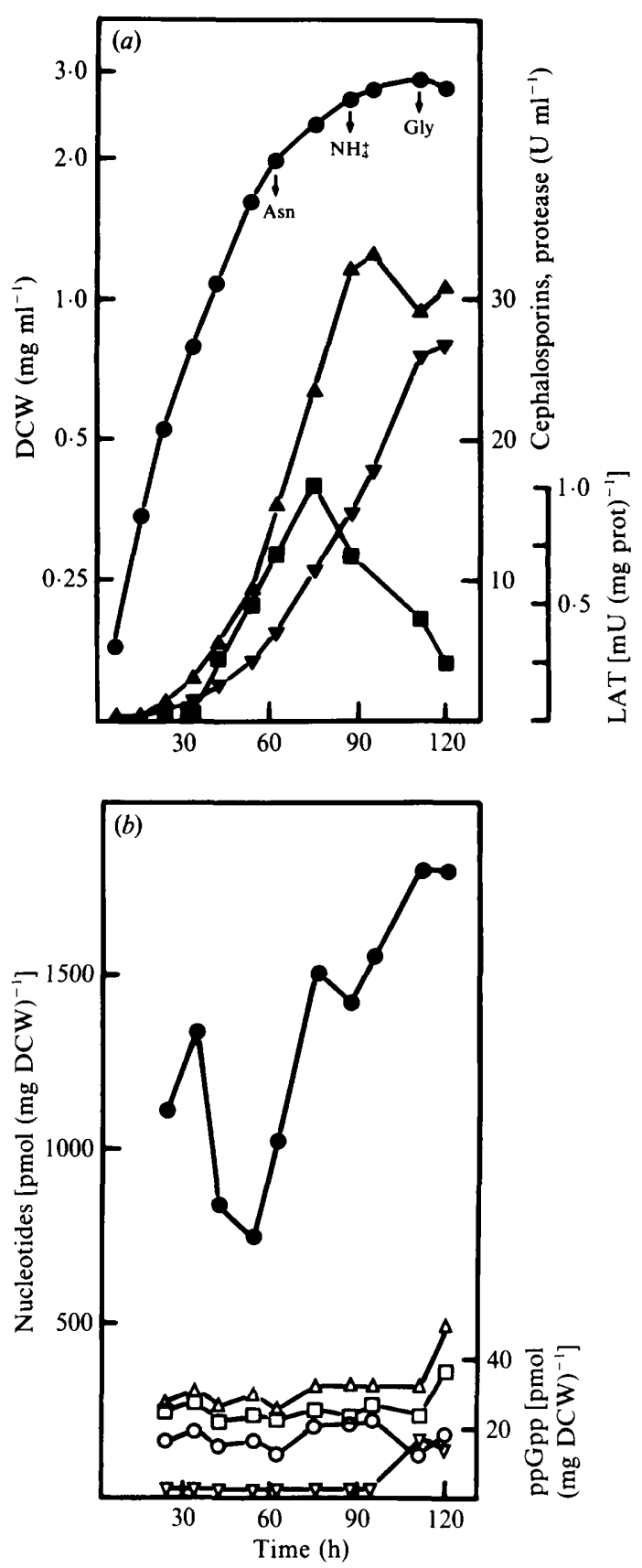

Fig. 1. Fermentation profiles during growth in MF medium with $15 \mathrm{~mm}$-asparagine. (a) Growth as dry cell weight $(\bullet)$, cephalosporins $(\boldsymbol{\Delta})$, extracellular protease $(\boldsymbol{\nabla})$ and lysine aminotransferase $(\boldsymbol{\square})$. The arrows indicate exhaustion of asparagine (Asn), ammonium $\left(\mathrm{NH}_{+}^{+}\right)$ and glycerol (Gly). (b) Intracellular concentrations of ATP (O), CTP $(\triangle)$, UTP $(\square)$, GTP $(O)$ and ppGpp $(\nabla)$.

Since well developed mycelium had been used as inoculum in the above experiment, it could be reasoned that initiation of secondary metabolism had been facilitated by previous initiation in the seed culture. Therefore, a similar experiment was performed using dormant spores $\left(2.4 \times 10^{5}\right.$ spores $\left.\mathrm{ml}^{-1}\right)$ as the inoculum. 

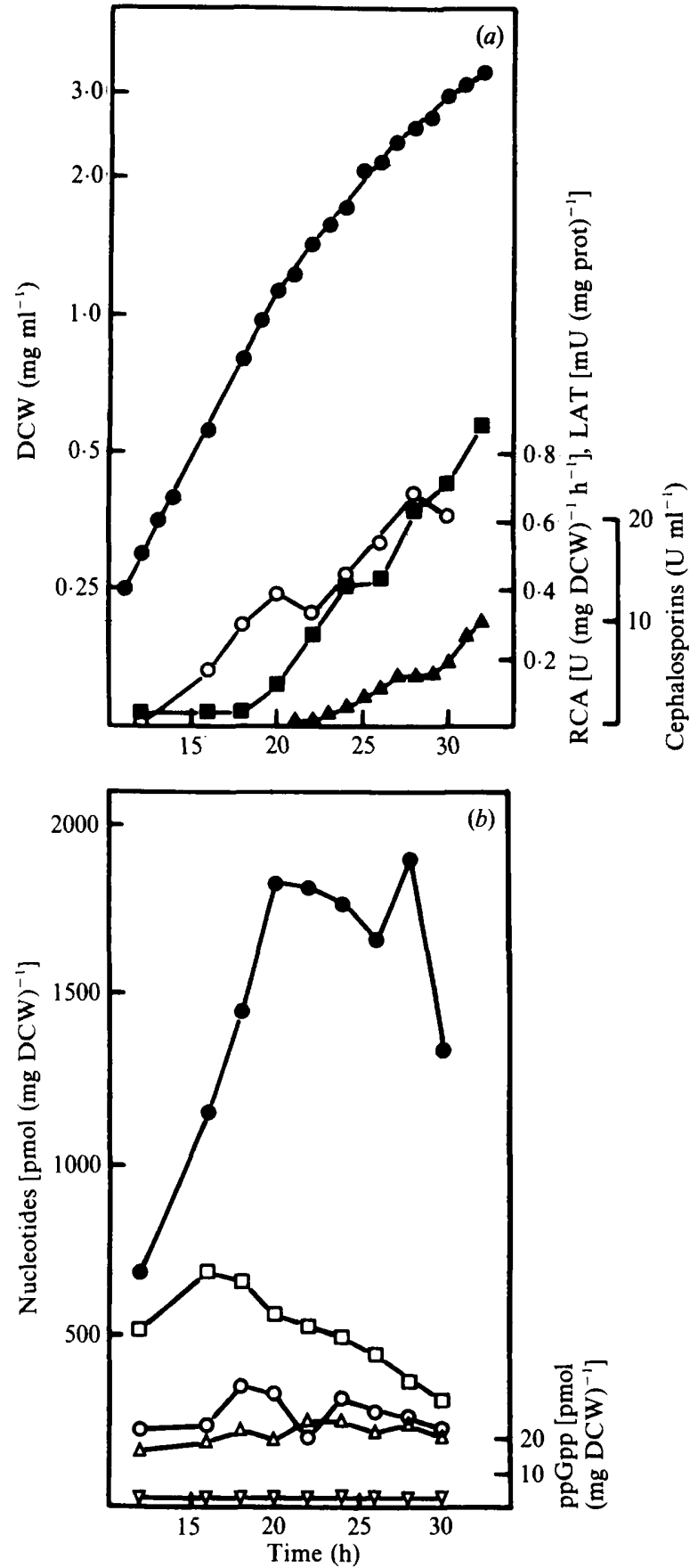

Fig. 2. Initiation of cephalosporin biosynthetic activity in MF medium with $15 \mathrm{~mm}$-asparagine and $1 \%(\mathrm{w} / \mathrm{v})$ Casamino acids. Vegetative mycelium was added as inoculum to give an initial cell density of $0.03 \mathrm{mg} \mathrm{ml}^{-1}$. (a) Dry cell weight (O), resting cell activity (O), lysine aminotransferase $(\boldsymbol{\square})$ and cephalosporins $(\boldsymbol{A})$. (b) Intracellular concentrations of ATP $(\circlearrowleft)$, UTP $(\square)$, GTP $(O)$, CTP $(\triangle)$ and ppGpp $(\nabla)$

The results were similar to those obtained with cultures inoculated with mycelium. The most remarkable difference was a more rapid increase in $\mathrm{RCA}$, which reached a maximum at a DCW close to $1 \mathrm{mg} \mathrm{ml}^{-1}$ compared to $2.7 \mathrm{mg} \mathrm{ml}^{-1}$ when the mycelial inoculum was used. Once more, ATP showed more dramatic changes in pool size than the other nucleotides, and ppGpp remained at basal levels (not shown).

\section{Stringent response in $S$. clavuligerus}

When cells growing in MF medium with asparagine and Casamino acids were transferred to the same medium without Casamino acids, the composition of their intracellular pools of nucleotides changed rapidly (Fig. $3 a, b$ ). ppGpp concentration peaked within $20 \mathrm{~min}$ of the shift-down. Maximum pppGpp levels [30 pmol (mg $\mathrm{DCW})^{-1}$ ] were observed at $10 \mathrm{~min}$, becoming undetectable in subsequent samples (not shown). These changes were transient, and values close to the initial ones were reached after $2 \mathrm{~h}$. In a similar experiment where asparagine was replaced by $\mathrm{NH}_{4} \mathrm{Cl}$ (Fig. 4), the ppGpp synthesizing ability of the cells upon Casamino acids deprivation was very low in young cultures, but increased markedly during early growth The use of $\mathrm{NH}_{4} \mathrm{Cl}$ instead of asparagine decreased RCA values, but did not modify the timing of initiation (compare with Fig. 2). In less detailed experiments in MF medium with asparagine and $1 \%$ Casamino acids, the ppGpp formation induced by Casamino acids deprivation was also extremely low in young mycelium and increased with growth (not shown).

RNA synthesis was under stringent control after nutritional shift-down from complex medium (Fig. 5). A similar decrease in RNA accumulation was obtained when DL-serine hydroxamate $\left(2.5 \mathrm{mg} \mathrm{ml}^{-1}\right)$ was added to cells growing in $\mathrm{MF}$ medium with $30 \mathrm{mM}-\mathrm{NH}_{4} \mathrm{Cl}$ as the only nitrogen source (not shown).

\section{Thiostrepton-resistant mutants}

Fifty-one spontaneous thiostrepton-resistant mutants were isolated in three independent experiments. All could grow and sporulate on sporulation medium supplemented with $5 \mu \mathrm{g}$ thiostrepton $\mathrm{ml}^{-1}$, although the lag phase was increased in most cases. In a preliminary screening in liquid MF medium with $30 \mathrm{~mm}$-proline and $1 \%(\mathrm{v} / \mathrm{v})$ Casamino acids, nine strains did not produce antibiotic. Two of these strongly acidified the medium and were not investigated further. The remaining seven (M604, M605, M611, M612, M616, M618 and M633) plus twenty other thiostrepton-resistant mutants were studied in more detail. Fig. 6 shows the cephalosporin titres obtained in complex medium (MF medium with TSB) plotted against the ppGpp produced upon nutritional shift-down in these twenty-seven mutants. There was great variability between strains, although all produced less ppGpp than the wild-type. The seven strains first characterized as non-producers maintained 

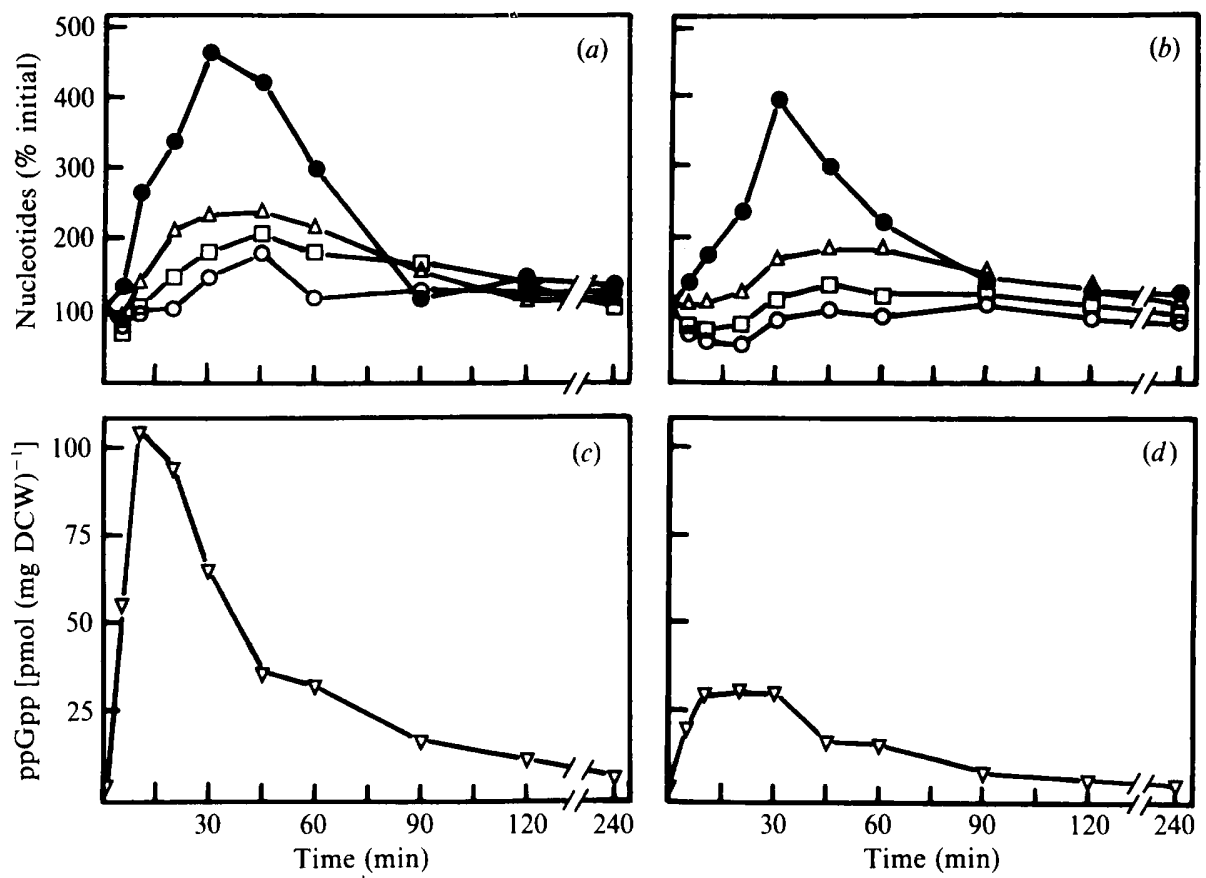

Fig. 3. Changes in the intracellular concentration of nucleotides in the wild type $(a, c)$ and mutant M604 $(b, d)$ after shift-down from MF medium with $15 \mathrm{~mm}$-asparagine and $1 \%(\mathrm{w} / \mathrm{v})$ Casamino acids to the same medium without Casamino acids. Cultures had a DCW of $1.2 \mathrm{mg} \mathrm{ml}^{-1}$ (wild type) or $1.0 \mathrm{mg} \mathrm{ml}^{-1}$ (M604) at the time of transfer, and were resuspended at the same cell density. Concentrations of nucleoside triphosphates are given as percentages of the values immediately before transfer. These values [pmol $\left.\left(\mathrm{mg} \mathrm{DCW}^{-1}\right)\right]$ were: 1170 (ATP), 295 (CTP), 386 (UTP) and 174 (GTP) for the wild-type; 1167 (ATP), 333 (CTP), 396 (UTP) and 253 (GTP) for M604.

Symbols as in Fig. 2.

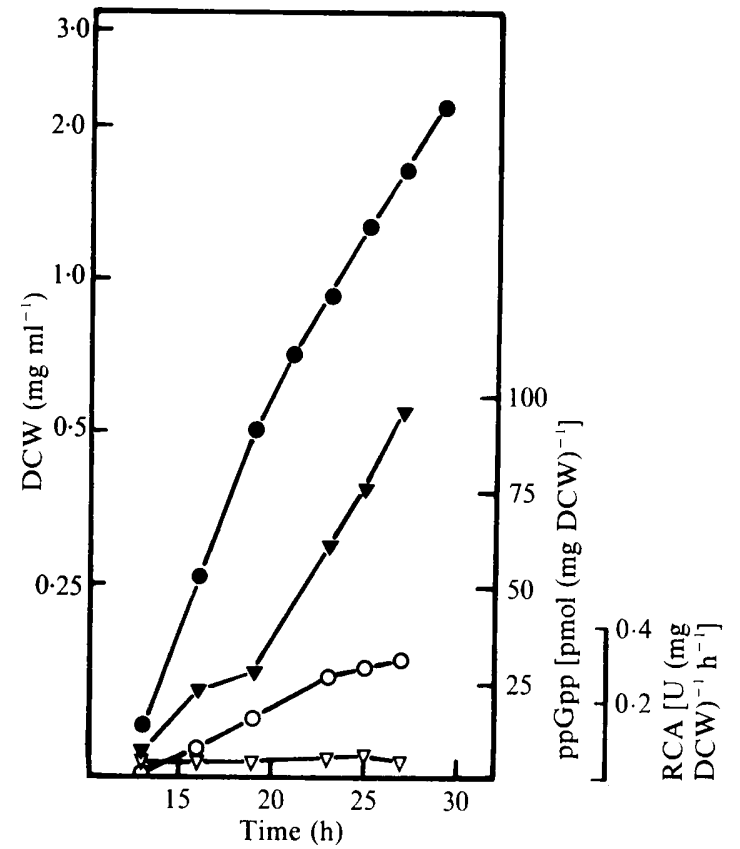

Fig. 4. Initiation of cephalosporin biosynthetic activity and changes in ppGpp-forming ability in $\mathrm{MF}$ medium with $30 \mathrm{mM}^{-\mathrm{NH}_{4}} \mathrm{Cl}$ and $1 \%$ (w/v) Casamino acids. Vegetative mycelium was added as inoculum to give an initial DCW of $0.02 \mathrm{mg} \mathrm{ml}^{-1}$. At intervals, a sample of the culture was transferred to the same medium without Casamino acids. The intracellular concentration of ppGpp was measured before $(\nabla)$ or 15 min after Casamino acids deprivation $(\nabla)$. Other symbols as in Fig. 2.

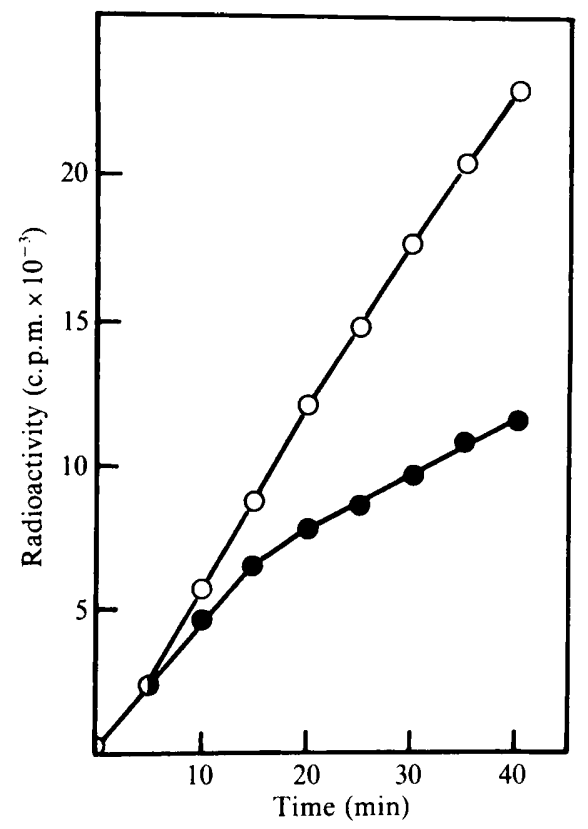

Fig.5. Effect of Casamino acids deprivation on RNA accumulation

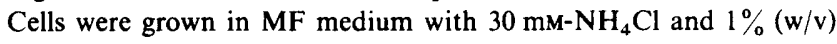
Casamino acids. At a DCW of $1.1 \mathrm{mg} \mathrm{m}^{-1}$, the mycelium was collected by filtration and transferred to $\mathrm{MF}$ medium containing $30 \mathrm{mM}-\mathrm{NH}_{4} \mathrm{Cl}$ and $20 \mu \mathrm{M}-\left[2-{ }^{14} \mathrm{C}\right]$ uracil, with $(\mathrm{O})$ or without $(O) 1 \%$ (w/v) Casamino acids. 


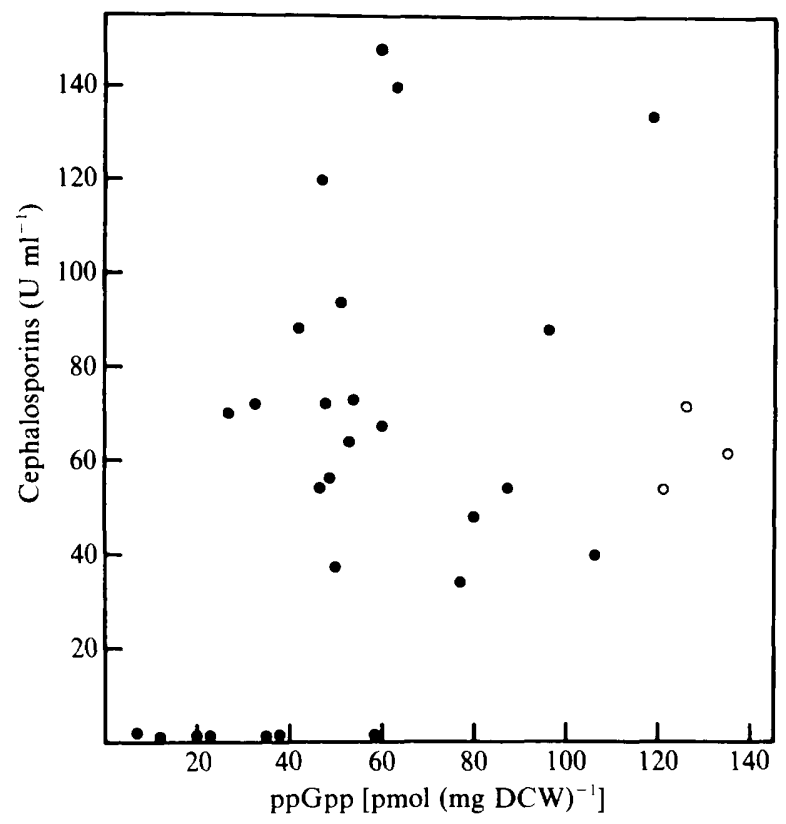

Fig. 6. Cephalosporin production and ppGpp formation in wild-type $(O)$ and thiostrepton-resistant mutants $(\Theta)$. Cephalosporin titres are the maximum values recorded in MF medium with $3 \%(w / v)$ TSB ppGpp was determined 10 and 20 min after a nutritional shift-down from $\mathrm{MF}$ medium containing $30 \mathrm{mM}-\mathrm{NH}_{4} \mathrm{Cl}$ and $1 \%(\mathrm{w} / \mathrm{v})$ Casamino acids to the same medium without Casamino acids. The higher of the two values obtained for each strain was used. Cultures were shifted down when growth had reached $0.8 \pm 0.1 \mathrm{mg} \mathrm{DCW} \mathrm{m}^{-1}$. Each closed circle represents a single mutant strain, whereas the open circles are the results obtained in three independent experiments with the wild-type.

this phenotype in this medium, while four of the other mutants had a high cephalosporin titre.

The reduced formation of ppGpp in the nonproducing mutants was not due to different kinetics of ppGpp synthesis, which were similar to those observed for the wild-type (a detailed experiment with mutant M604 is shown in Fig. 3c,d) Furthermore, when ppGpp formation was induced by Casamino acids deprivation at different growth stages (in an experiment similar to that illustrated in Fig. 4), M604 always had ppGpp pools lower than $30 \%$ of the corresponding ones in the wild type (not shown). Accumulation of RNA after nutritional shift-down was tested in six of the seven nonproducing mutants. In all of them, RNA synthesis was partially inhibited, as observed for the wild-type (not shown).

To assess the significance of the large differences in cephalosporin synthesis shown in Fig. 6, thirty sporulated colonies were taken at random from wild-type plates and screened individually for production of antibiotic and extracellular protease in complex medium (Fig. 7). Considerable variation was found among wild-type isolates, but all of them were producers. Those thiostrep-
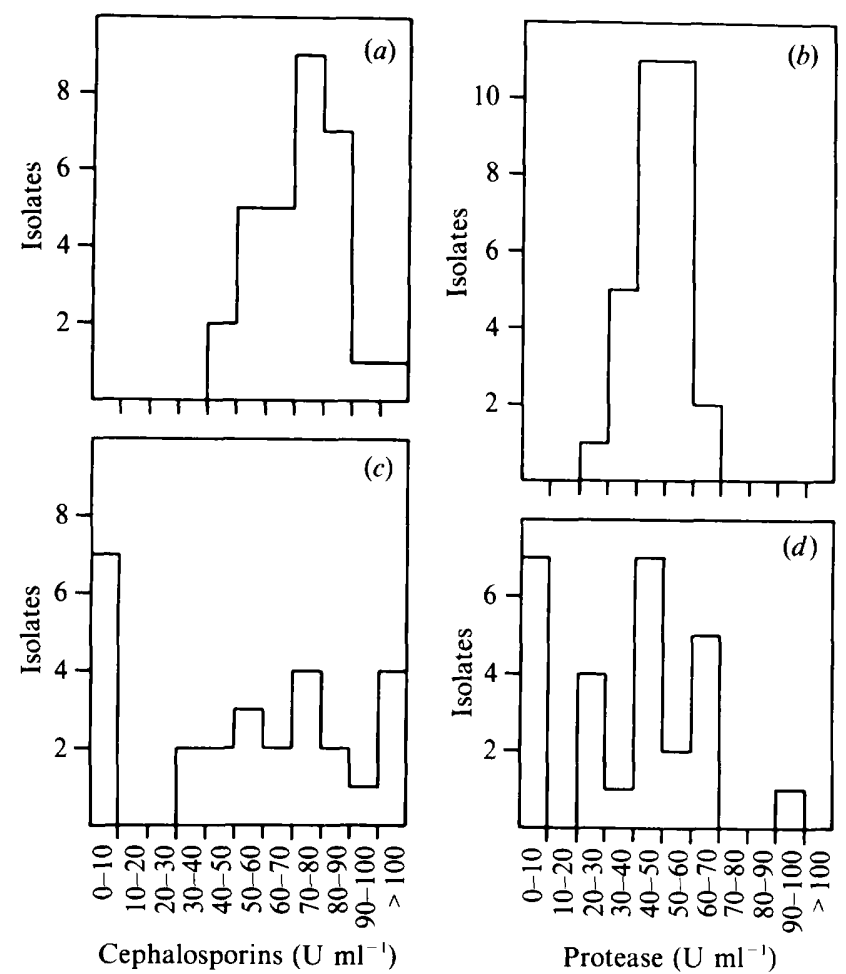

Fig. 7. Production of cephalosporins $(a, c)$ and extracellular protease $(b, d)$ in wild-type isolates $(a, b)$ and thiostrepton-resistant mutants $(c$, d). Maximum values recorded in MF medium with $3 \%$ TSB are shown. Cephalosporin titres of the mutants are taken from Fig. 6 . The highest cephalosporin titre recorded for a wild-type isolate was $104 \mathrm{U} \mathrm{ml}^{-1}$.

ton-resistant mutants that failed to produce cephalosporins also had very low protease titres.

The effect of these thiostrepton resistance mutations on growth and secondary metabolism was tested in different media. Several mutants were examined, but the most detailed study was carried out with strain M633 (Table 2). Its behaviour was strongly dependent on the nitrogen source used in the medium. In defined MF medium containing a single nitrogen source such as asparagine, glutamine or $\mathrm{NH}_{4} \mathrm{Cl}$, that provided nitrogen-excess conditions (i.e. repressed levels of glutamine synthetase) (Bascarán et al., 1989), growth was normal and the maximum values of cephalosporins and protease were somewhat lower than those of the wild-type. With arginine, alanine or glutamate, amino acids that provide nitrogen-limiting conditions (i.e. derepressed levels of glutamine synthetase; Bascarán et al., 1989), and with complex nitrogen sources, both the rate and extent of growth were poorer, and production of antibiotic and protease was very low. Although examined in less detail, the other six mutants that did not produce in MF medium with TSB, showed the same type of behaviour as strain M633 when grown in media of the above three 
Table 2. Growth and secondary metabolism in the wild type and mutant M633 as a function of the nitrogen source

Cultures were grown in MF medium with the indicated nitrogen sources and sampled twice a day until DCW decreased in two consecutive determinations. Growth and production values are the maximum values recorded during the fermentations.

\begin{tabular}{|c|c|c|c|c|c|c|}
\hline \multirow[b]{3}{*}{ Nitrogen source* } & \multicolumn{6}{|c|}{ Strain } \\
\hline & \multicolumn{3}{|c|}{ Wild-type } & \multicolumn{3}{|c|}{ M633 } \\
\hline & $\begin{array}{c}\text { DCW } \\
\left(\mathrm{mg} \mathrm{ml}^{-1}\right)\end{array}$ & $\begin{array}{l}\text { Cephalosporins } \\
\left(\mathrm{U} \mathrm{ml}^{-1}\right)\end{array}$ & $\begin{array}{l}\text { Protease } \\
\left(\mathrm{U} \mathrm{ml}^{-1}\right)\end{array}$ & $\begin{array}{c}\text { DCW } \\
\left(\mathrm{mg} \mathrm{ml}^{-1}\right)\end{array}$ & $\begin{array}{l}\text { Cephalosporins } \\
\left(\mathrm{U} \mathrm{ml}^{-1}\right)\end{array}$ & $\begin{array}{l}\text { Protease } \\
\left(\mathrm{U} \mathrm{ml}^{-1}\right)\end{array}$ \\
\hline Arginine & $2 \cdot 6$ & $7 \cdot 5$ & $19 \cdot 2$ & $1 \cdot 3$ & 1.4 & $1 \cdot 1$ \\
\hline Alanine & 3.9 & $17 \cdot 8$ & $42 \cdot 1$ & 1.0 & $<1.0$ & 0.4 \\
\hline Glutamate & 3.9 & 20.9 & $36 \cdot 7$ & $3 \cdot 0$ & $1 \cdot 2$ & $0 \cdot 3$ \\
\hline $\mathrm{NH}_{4} \mathrm{Cl}$ & $3 \cdot 7$ & $12 \cdot 5$ & $4 \cdot 2$ & 3.9 & $5 \cdot 8$ & 1.6 \\
\hline Glutamine & 3.5 & $30 \cdot 0$ & $40 \cdot 1$ & $3 \cdot 3$ & $26 \cdot 8$ & $20 \cdot 5$ \\
\hline Asparagine & $3 \cdot 6$ & $33 \cdot 0$ & $32 \cdot 0$ & 3.5 & $22 \cdot 7$ & $15 \cdot 7$ \\
\hline Casamino acids & $7 \cdot 3$ & 49.5 & $29 \cdot 7$ & $2 \cdot 5$ & $<1.0$ & $2 \cdot 1$ \\
\hline Yeast extract & 8.6 & $27 \cdot 3$ & $59 \cdot 4$ & 1.4 & $<1.0$ & $2 \cdot 6$ \\
\hline TSB & $10 \cdot 5$ & $66 \cdot 0$ & $49 \cdot 6$ & 3.7 & $1 \cdot 4$ & $3 \cdot 3$ \\
\hline
\end{tabular}

* Amino acids and $\mathrm{NH}_{4} \mathrm{Cl}$ were used at a concentration providing 30 mM-nitrogen; Casamino acids and yeast extract were used at $1 \%(\mathrm{w} / \mathrm{v})$, and TSB was used at $3 \%(\mathrm{w} / \mathrm{v})$.

Table 3. Extracellular protease production after nutritional shift-down in the wild-type and mutant M633

Cells grown in MF medium with $15 \mathrm{mM}$-asparagine and $1 \%$ Casamino acids were transferred to MF medium with the indicated nitrogen sources at a DCW of $1.0 \mathrm{mg} \mathrm{ml}^{-1}$ and incubated for $8 \mathrm{~h}$

\begin{tabular}{lcc}
\hline \hline & \multicolumn{2}{c}{ Protease $\left(\mathrm{U} \mathrm{ml}^{-1}\right)$} \\
\cline { 2 - 3 } Nitrogen source* & Wild-type & $\mathrm{M} 633$ \\
\hline None & 3.91 & 0.58 \\
Proline & 2.00 & 0.43 \\
Arginine & 1.15 & 0.40 \\
Alanine & 0.60 & 0.37 \\
Yeast extract & 5.73 & 0.24 \\
\hline \hline
\end{tabular}

* Amino acids were used at a concentration providing $30 \mathrm{mM}$ nitrogen; yeast extract was used at $0 \cdot 1 \%(\mathrm{w} / \mathrm{v})$.

classes. Other thiostrepton-resistant mutants that produced normal (M621 and M628) or high (M602 and M631) levels of cephalosporins in MF medium with TSB were studied more carefully in that medium and in MF medium with asparagine. In the first medium, all four mutants grew slightly slower than the wild-type (initial doubling times were $2.3 \mathrm{~h}$ for the wild-type and $2 \cdot 9-4 \cdot 1 \mathrm{~h}$ for the mutants), but reached similar maximum biomass values. In MF medium with asparagine they were indistinguishable in growth and production of antibiotic and protease from the wild-type (not shown).

Although cephalosporin synthesis by the wild-type strain was initiated in complex media without any need for nutritional shift-down, no extracellular protease was detected during early growth under these conditions. Shift-down to nitrogen-limiting media initiated protease production in the wild-type, as previously reported (Bascarán et al., 1990), but not in mutant M633 (Table 3).

\section{Discussion}

Several types of evidence indicate that LAT activity is linked to the $\beta$-lactam biosynthetic pathway in $S$. clavuligerus. Among several Streptomyces species tested, it was found only in $\beta$-lactam producers but did not allow $S$. clavuligerus to grow on lysine as sole nitrogen source (Madduri et al., 1989). Furthermore, its levels were altered in some $S$. clavuligerus mutants with deficiencies in antibiotic production (Romero et al., 1988). Our results also suggest that the degradative pathway involving CAT is used to catabolize lysine as a nitrogen source (Madduri et al., 1989) and may be partially under nitrogen control. LAT was highest in media supporting relatively good cephalosporin production, and its activity profile in defined medium was similar to those reported for isopenicillin $\mathrm{N}$ synthase and deacetoxycephalosporin C synthase (Braña et al., 1985). Therefore, this enzyme may be a useful indicator for secondary metabolism since, unlike other cephalosporin biosynthetic enzymes, it can be assayed with a readily available substrate.

We followed two approaches to investigate the possible role of the stringent response in the initiation of 
two secondary metabolic activities; the biosynthesis of cephalosporins and of extracellular protease. Cephalosporin biosynthesis was assessed not only as LAT activity and cephalosporin production but also by using RCA as an 'in vivo' measure of the functioning of the whole pathway. In the first approach, we measured nucleotide pools during initiation of cephalosporin synthesis. In all conditions tested, initiation took place while ppGpp remained stable at basal levels. The existence of a missing ppGpp peak in this period is unlikely, since the cultures were sampled frequently and the young mycelium had a very low ppGpp-producing ability. The latter characteristic had been also reported in Streptomyces antibioticus (Ochi, 1987b). The intracellular concentration of the other nucleotides measured in $S$. clavuligerus were similar to those reported for Streptomyces hygroscopicus (Riesenberg et al., 1984). The rapid increase in the ATP pool during initiation of antibiotic synthesis may be of interest, since it indicates that the cells were changing during exponential growth. The only circumstance in which a slightly higher ppGpp pool was found during growth was in defined media with nitrogen sources (such as proline or arginine) that supported low growth rates. Such conditions do not favour formation of cephalosporin biosynthetic enzymes (Braña et al., 1986) or protease production (Bascarán et al., 1990). Although initiation of antibiotic synthesis is not frequently observed during rapid growth in rich media, a sharp derepression of isopenicillin $\mathrm{N}$ synthase and deacetoxycephalosporin $\mathrm{C}$ synthase has been described in $S$. clavuligerus during early growth in a rich medium containing TSB (Rollins et al., 1990), a result consistent with our observations using resting cell systems.

The second approach we followed was the isolation and characterization of thiostrepton-resistant mutants. Although marked differences were observed between individual mutants, most had a significantly diminished capacity to produce ppGpp upon nutritional shift-down. However, on the basis of antibiotic production in rich medium, three classes of mutants could be distinguished: the first produced very little antibiotic or protease; the second, to which most of the mutants belonged, gave antibiotic titres within the range obtained with the wild type; the third class gave cephalosporin titres higher than the wild type, although their protease production was normal. Thus, it seems that the mutations conferring thiostrepton-resistance can influence cephalosporin production in opposite ways, depressing or stimulating the process, and the effect is partially pleiotropic, affecting protease synthesis in the first class of mutants. Since a wide range of ppGpp formation was found within each class, no correlation could be established between the secondary metabolic traits studied and the functioning of the stringent response. In their severely reduced synthesis of ppGpp and depressed secondary metabolism, mutants of the first class resemble the thiostreptonresistant mutants described by Ochi in other Streptomyces species (Ochi, 1986, 1987a, b, 1988). However, mutants with the characteristics of the other two classes were not reported.

Thiostrepton is a protein synthesis inhibitor that binds to the 50S ribosomal subunit and interferes with a series of activities involving GTP, including the formation of (p)ppGpp dependent upon stringency-factor (Cundliffe, 1986). Spontaneous thiostrepton-resistant mutants of Bacillus species have been found to lack ribosomal protein L11 (Cundliffe et al., 1979; Wienen et al., 1979). These strains, as well as other $E$. coli mutants with an altered L11 protein (Parker et al., 1976), grow poorly in various media, have less ability to produce ppGpp and exhibit a relaxed phenotype (relC mutants; Smith et al., 1978). These characteristics resemble those reported for thiostrepton-resistant mutants of several Streptomyces species (Ochi, 1990). They also resemble in part those found in the first class of thiostrepton-resistant mutants described in this work. However, stringent control of RNA accumulation, although relatively weak in all cases, was maintained in our mutants. This may be due to their residual production of $\mathrm{ppGpp}$ or to the existence of another mechanism of stringency not involving ppGpp, as found in other bacteria (Spadaro et al., 1981; Belitsky \& Kari, 1982; Acosta \& Lueking, 1987; McDowell et al., 1988). The poor growth of this class of $S$. clavuligerus mutants could be corrected in defined media with a good single nitrogen source. The fact that secondary metabolic activities were partially recovered under these conditions suggests that the deficiencies in secondary metabolism are somehow linked to abnormal growth, probably due to a ribosomal alteration. It should be noted, however, that secondary metabolism was affected more severely than growth in the first class of mutants, and cephalosporin production was enhanced in the third class, even though growth was also slower in rich medium. Therefore, the alterations observed in secondary metabolism do not appear to be a simple consequence of differences in growth rate or extent, but may be related to the availability of precursors for ribosomal activity.

Our results do not rule out a possible stimulating effect of ppGpp on antibiotic production, but they show that there is no obligate relationship between this activity and the stringent response in $S$. clavuligerus. Initiation of most antibiotic biosynthetic processes requires suboptimal growth conditions imposed by some nutritional limitation (Martin \& Demain, 1980). Thus, initiation of synthesis following a nutritional shift-down, as observed in other species (Ochi, 1986, 1987a, $b, 1988$ ), may be 
a consequence of the transfer to non-repressing conditions, rather than a result of the transient production of ppGpp. This may also be true for the initiation of protease synthesis in $S$. clavuligerus after a nutritional shift-down to defined media with poor nitrogen sources. The impairment in antibiotic synthesis observed in thiostrepton-resistant mutants could be explained by a ribosomal alteration that interferes with ppGpp formation and also critically affects secondary metabolism initiation by a mechanism not involving ppGpp. A precedent for the regulatory effect of a relC mutation, independent of ppGpp formation, has been described for the synthesis of a chloramphenicol acetyltransferase in Bacillus subtilis (Ambulos et al., 1988; Lovett, 1990). The isolation of relaxed mutants of Streptomyces griseus that had recovered their antibiotic production ability has been recently reported (Ochi, 1990). The new phenotype was attributed to a second mutation at a suppressor locus, but may also indicate the lack of a causal relationship between the stringent response and secondary metabolism. Other recent observations show that initiation of antibiotic synthesis in Streptomyces coelicolor is not necessarily coupled to ppGpp synthesis (Strauch et al., 1991). Furthermore, the structure and function of Streptomyces ribosomes change markedly during the complex developmental cycle of these organisms (Quirós et al., 1989; L. M. Quirós and coworkers, unpublished results). The dramatic increase observed in ppGpp-forming ability in the course of secondary metabolism initiation in our work suggests the possible existence of changes in ribosome-related functions that are crucial for that initiation and can be perturbed in thiostrepton-resistant mutants. Such ribosomal functions may participate in the hierarchical control of secondary metabolism. The nutritional status of the cells would determine the degree of expression of particular pathways. Thus, in $S$. clavuligerus, cephalosporin synthesis is favoured by good nutritional conditions and can take place during early growth in different media (Braña et al., 1986; Vining et al., 1987; this work), whereas a lower nitrogen supply (or growth rate) is required for optimal formation of protease (Bascarán $e t$ al., 1990; this work).

V. Bascarán received a grant from the Plan de Formación de Personal Investigador of the Ministry of Education of Spain.

\section{References}

Acosta, R. \& Lueking, D. R. (1987). Stringency in the absence of ppGpp accumulation in Rhodobacter sphaeroides. Journal of Bacteriology 169, 908-912.

Adamidis, T., Riggle, P. \& ChampNess, W. (1990). Mutations in a new Streptomyces coelicolor locus which globally block antibiotic biosynthesis but not sporulation. Journal of Bacteriology 172, 2962-2969.
Aharonowitz, Y. \& Demain, A. L. (1977). Influence of inorganic phosphate and organic buffers on cephalosporin production by Streptomyces clavuligerus. Archives of Microbiology 115, 169-173.

Aharonowitz, Y. \& Demain, A. L. (1979). Nitrogen nutrition and regulation of cephalosporin production in Streptomyces clavuligerus. Canadian Journal of Microbiology 25, 61-67.

Ambulos, N. P., Rogers, E. J., Alexieva, Z. \& Lovett, P. S. (1988). Induction of cat -86 by chloramphenicol and amino acid starvation in relaxed mutants of Bacillus subtilis. Journal of Bacteriology 170, 56425646.

AN, G. \& VINING, L. C. (1978). Intracellular levels of guanosine 5'diphosphate $3^{\prime}$-diphosphate (ppGpp) and guanosine 5'-triphosphate $3^{\prime}$-diphosphate (pppGpp) in cultures of Streptomyces griseus producing streptomycin. Canadian Journal of Microbiology 24, 502-511.

Bascarán, V., Hardisson, C. \& Braña, A. F. (1989). Regulation of nitrogen catabolic enzymes in Streptomyces clavuligerus. Journal of General Microbiology 135, 2465-2474.

Bascarán, V., Hardisson, C. \& Braña, A. F. (1990). Regulation of extracellular protease production in Streptomyces clavuligerus. Applied Microbiology and Biotechnology 34, 208-213.

BeLITSKY, B. \& KARI, C. (1982). Absence of accumulation of ppGpp and RNA during amino acid starvation in Rhizobium meliloti. Journal of Biological Chemistry 257, 4677-4679.

BoK, S. H. \& Demain, A. L. (1977). An improved assay for polyols. Analytical Biochemistry 81, 18-20.

Braña A. F., Wolfe, S. \& Demain, A. L. (1985). Ammonium repression of cephalosporin production by Streptomyces clavuligerus. Canadian Journal of Microbiology 31, 736-743.

Braña, A. F., Wolfe, S. \& Demain, A. L. (1986). Relationship between nitrogen assimilation and cephalosporin synthesis in Streptomyces clavuligerus. Archives of Microbiology 146, 46-51.

Bushell, M. E. \& FrYDAY, A. (1983). The application of materials balancing to the characterization of sequential secondary metabolite formation in Streptomyces cattleya NRRL 8057. Journal of General Microbiology 129, 1733-1741.

CUNDLIFFE, E. (1986). Involvement of specific portions of ribosomal RNA in defined ribosomal functions: a study utilizing antibiotics. In Structure, Function, and Genetics of Ribosomes, pp. 586-604. Edited by B. Hardesty \& G. Kramer. New York: Springer-Verlag.

Cundliffe, E., Dixon, P., Stark, M., Stöffler, G., Ehrlich, R., StÖFfleR-MeILICKE, M. \& CANNON, M. (1979). Ribosomes in thiostrepton-resistant mutants of Bacillus megaterium lacking a single 50 S subunit protein. Journal of Molecular Biology 132, 235-252.

Freese, E., OlempSKa-BeEr, Z. \& Eisenberg, M. (1984). Nucleotide composition of cell extracts analysed by full-spectrum recording in high-performance liquid chromatography. Journal of Chromatography 284, 125-142.

Freese, E., Freese, E. B., Allen, E. R., Olempska-Beer, Z., Orrego, C., VARMA, A. \& WABIKo, H. (1985). Metabolic initiation of spore development. In Molecular Biology of Microbial Differentiation, pp. 194-202. Edited by J. A. Hoch \& P. Setlow. Washington DC: American Society for Microbiology.

GINTHER, C. L. (1979). Sporulation and the production of serine protease and cephamycin C by Streptomyces lactamdurans. Antimicrobial Agents and Chemotherapy 15, 522-526.

Hardisson, C., Manzanal, M. B., Salas, J. A. \& Suarez, J. E. (1978). Fine structure, physiology and biochemistry of arthrospore germination in Streptomyces antibioticus. Journal of General Microbiology 105, 203-214.

Hill, D. W., Walters, F. M., Wilson, D. \& Stuart, J. D. (1979). High performance liquid chromatographic determination of amino acids in the picomole range. Analytical Chemistry 51, 1338-1341.

Hobbs, G., Frazer, C. M., Gardner, D. C. J., Flett, F. \& Oliver, S. G. (1990). Pigmented antibiotic production by Streptomyces coelicolor A3(2): kinetics and the influence of nutrients. Journal of General Microbiology 136, 2291-2296.

HopwOOD, D. A. (1988). Understanding the genetic control of antibiotic biosynthesis and sporulation in Streptomyces. In Biology of Actinomycetes '88, pp. 3-10. Edited by Y. Okami, T. Beppu \& H. Ogawara. Tokyo: Japan Scientific Societies Press.

LOVETT, P. S. (1990). Translational attenuation as the regulator of inducible cat genes. Journal of Bacteriology 172, 1-6. 
McDowell, T. D., Reed, K. E. \& Hadley, W. M. (1988). Accumulation of ppGpp in three streptococci during periods of amino acid starvation. FEMS Microbiology Letters 56, 151-156.

Madduri, K., StutTaRd, C. \& Vining, L. C. (1989). Lysine catabolism in Streptomyces spp. is primarily through cadaverine: $\beta$-lactam producers also make $\alpha$-aminoadipate. Journal of Bacteriology 171, 299-302.

Martin, J. F. \& Demain, A. L. (1980). Control of antibiotic biosynthesis. Microbiological Reviews 44, 230-251.

OCHI, K. (1986). Occurrence of the stringent response in Streptomyces $\mathrm{sp}$. and its significance for the initiation of morphological and physiological differentiation. Journal of General Microbiology 132, 2621-2631.

OCHI, K. (1987a). Metabolic initiation of differentiation and secondary metabolism by Streptomyces griseus: significance of the stringent response (ppGpp) and GTP content in relation to A factor. Journal of Bacteriology 169, 3608-3616.

OCHI, K. (1987b). A rel mutation abolishes the enzyme induction needed for actinomycin synthesis by Streptomyces antibioticus. Agricultural and Biological Chemistry 51, 829-835.

OCHI, K. $(1987 c)$. Changes in nucleotide pools during sporulation of Streptomyces griseus in submerged culture. Journal of General Microbiology 133, 2787-2795.

OCHI, K. (1988). Nucleotide pools and stringent response in regulation of Streptomyces differentiation. In Biology of Actinomycetes '88, pp. 330-337. Edited by Y. Okami, T. Beppu \& H. Ogawara. Tokyo: Japan Scientific Societies Press.

OCHI, K. (1990). Streptomyces relC mutants with an altered ribosomal protein ST-L11 and genetic analysis of a Streptomyces griseus relC mutant. Journal of Bacteriology 172, 4008-4016.

OChI, K., Kandala, J. C. \& Freese, E. (1981). Initiation of Bacillus subtilis sporulation by the stringent response to partial amino-acid deprivation. Journal of Biological Chemistry 256, 6866-6875.

Parker, J., Watson, R. J. \& Friesen, J. D. (1976). A relaxed mutant with an altered ribosomal protein L11. Molecular and General Genetics 144, 111-114.

Quirós, L. M., Parra, F., Hardisson, C. \& Salas, J. A. (1989). Structural and functional analysis of ribosomal subunits from vegetative mycelium and spores of Streptomyces antibioticus. Journal of General Microbiology 135, 1661-1670.
RiesenberG, D., Bergter, F. \& Kari, C. (1984). Effect of serine hydroxamate and methyl $\alpha$-D-glucopyranoside treatment on nucleoside polyphosphate pools, RNA and protein accumulation in Streptomyces hygroscopicus. Journal of General Microbiology 130, 2549-2558.

Rollins, M. J., Jensen, S. E., Wolfe, S. \& Westlake, D. W. S. (1990). Oxygen derepresses deacetoxycephalosporin $C$ synthase and increases the conversion of penicillin $\mathbf{N}$ to cephamycin $\mathrm{C}$ in Streptomyces clavuligerus. Enzyme and Microbial Technology 12, 40-45.

Romero, J., Liras, P. \& MARTIN, J. F. (1984). Dissociation of cephamycin and clavulanic acid biosynthesis in Streptomyces clavuligerus. Applied Microbiology and Biotechnology 20, 318-325.

ROMERo, J., LIRAS, P. \& MARTIN, J. F. (1988). Isolation and biochemical characterization of Streptomyces clavuligerus mutants in the biosynthesis of clavulanic acid and cephamycin C. Applied Microbiology and Biotechnology 27, 510-516.

Šmúth, J., Hudec, J., Chau, H. T., DÁNyi, O. \& Zelinka, J. (1979). The synthesis of highly phosphorylated nucleotides, RNA and protein by Streptomyces aureofaciens. Journal of Antibiotics 32, 53-58.

Smith, I., Paress, P. \& Pestka, S. (1978). Thiostrepton-resistant mutants exhibit relaxed synthesis of RNA. Proceedings of the National Academy of Sciences of the United States of America 75, 5993-5997.

Spadaro, A., Spena, A., Santonastaso, V. \& Donini, P. (1981). Stringency without ppGpp accumulation. Nature, London 291, 256258.

Strauch, E., Takano, E., Baylis, H. A. \& Bibb, M. J. (1991). The stringent response in Streptomyces coelicolor A3(2). Molecular Microbiology 5, 289-298.

Vining, L. C., Jensen, S., Westlake, D. W. S., Aharonowitz, Y. \& WolfE, S. (1987). Cephamycin production and isopenicillin $N$ synthetase activity in cultures of Streptomyces clavuligerus. Applied Microbiology and Biotechnology 27, 240-246.

WEATHERBURN, M. W. (1967). Phenol-hypochlorite reaction for determination of ammonia. Analytical Chemistry 39, 971-974.

Wienen, B. Ehrlich, R., StöfFler-Meilicke, M., Stöffler, G., SMITH, I., Weiss, D., VinCE, R. \& PESTKA, S. (1979). Ribosomal protein alterations in thiostrepton- and micrococcin-resistant mutants of Bacillus subtilis. Journal of Biological Chemistry 254, 80318041. 